\title{
Aerodynamic Design Optimisation of Non-planar Lifting Surfaces
}

\author{
Shaun N. Skinner* and Hossein Zare-Betash ${ }^{\dagger}$ \\ School of Engineering, University of Glasgow, Scotland, United Kingdom, G12 8QQ
}

\begin{abstract}
A novel population structured genetic algorithm (sGA) with embedded potential flow vortex ring panel method (VRM) has been developed to minimise induced and parasitic drag subject to constraints on lift, root bending moment, and longitudinal static stability. The optimisation architecture can activate up to four independent wing segments allowing up to 28 design variables. Minimum drag of wing tip extensions and winglet configurations are compared using the non-linear stochastic optimisation method. The optimiser identified joined box wings as offering the greatest induced efficiency followed by C-wings. With span and root bending moment constraints winglets offered best total drag reduction. C-wings are further investigated for potential to enhance longitudinal static stability performance by staggering the horizontal extension of the winglet to balance moments around the wing's centre of gravity. Preliminary results suggest that while longitudinal static stability can be reached it would be very poor. Inclusion of more design constraints and additional analysis of the structural dynamics of C-wings, especially effecting the torsional mode, is necessary.
\end{abstract}

\section{Nomenclature}

$\begin{array}{ll}A R & \text { Aspect Ratio } \\ b & \text { Span of wing segment, } m \\ c & \text { Chord, } m \\ C_{D} & \text { Wing drag force coefficient } \\ c_{d} & \text { Sectional drag force coefficient } \\ C_{L} & \text { Wing lift force coefficient } \\ c_{l} & \text { Sectional lift force coefficient } \\ C_{l_{\alpha}} & \text { Lift-curve slope } \\ C_{M A C} & \text { Mean aerodynamic chord, } m \\ C_{m_{\alpha}} & \text { Pitch stiffness derivative } \\ D_{\text {ind }} & \text { Induced Drag, } N \\ D_{\text {para }} & \text { Parasitic Drag, } N \\ h_{\text {wingtip }} & \text { Maximum height of wing tip, } m \\ K_{n} & \text { Static Margin } \\ L & \text { Lift, } N \\ M_{\text {root }} & \text { Root bending moment, } N m^{-1} \\ m & \text { Root bending moment constraint coefficient } \\ N C & \text { Number of chord-wise aerodynamic panels } \\ N P & \text { Number of span-wise aerodynamic panels } \\ n & \text { Normal vector } \\ R e & \text { Reynolds number } \\ s & \text { Span constraint coefficient } \\ y & \text { Span-wise coordinate } \\ \bar{V}_{h} & \text { Horizontal tail volume coefficient } \\ X_{C G} & \text { Centre of gravity longitudinal position } \\ \end{array}$

${ }^{*}$ PhD Research Student, School of Engineering, University of Glasgow, and Student Member AIAA.

${ }^{\dagger}$ Lecturer, School of Engineering, University of Glasgow, and Senior Member AIAA. 


$\begin{array}{ll}\text { Subscript } & \\ i & \text { Variable number } \\ \text { ref } & \text { Reference } \\ \text { limit } & \text { Limit }\end{array}$

$\begin{array}{ll}\text { Symbols } & \\ \alpha & \text { angle of attack, degrees } \\ \Gamma & \text { Dihedral angle, degrees } \\ \gamma & \text { Span loading }\left[\frac{c_{l i} c_{i}}{C_{L} C_{M A C}}\right] \\ \theta & \text { Local twist angle, degrees } \\ \Lambda & \text { Mid-chord sweep angle, degrees } \\ \lambda & \text { Taper Ratio } \\ \rho & \text { Density, } \mathrm{kgm}^{-3} \\ \Delta \varphi & \text { Potential jump, } \mathrm{m}^{2} \mathrm{~s} \\ \nabla \varphi & \text { Potential velocity }\end{array}$

\section{Introduction}

NON-PLANAR lifting surfaces have been widely recognised as offering reductions in total drag when compared to conventional planar wing systems of the same span and lift. ${ }^{1}$ In the cruise phase of large transport aircraft, typically $90 \%$ of total flight time ${ }^{2}$ drag consists of parasitic and induced drag; the induced drag is relatively lower than the parasitic drag but still contributes $40 \%-45 \%$ of the total drag budget. ${ }^{2,3}$ A $1 \%$ reduction of the total drag for an A340 aircraft operating in long range mode saves 400,000 litres of fuel and consequently $5000 \mathrm{~kg}$ of noxious emissions per year, also indirectly reducing noise. ${ }^{4}$ Thus, as a consequence of seeking better fuel burn the trend in the wing design of transport aircraft has been to increase the aspect ratio to improve the lift-to-drag ratio. ${ }^{5,6}$ This trend is driven by increases in fuel prices.

Non-planar wing tip geometry can be manipulated to strongly affect induced drag; wing tip optimisation has received much attention over recent years however still remains an area of research with little consensus. As a result numerous non-planar wing tip devices have been developed, some of the most successful (Whitcomb's winglet, ${ }^{7}$ tipsails, ${ }^{8}$ Lockheed Martin's vortex diffuser, ${ }^{9}$ Airbus' A310 tip fence, ${ }^{10}$ A330/A340 winglet, ${ }^{11}$ and the 'sharklet'11,12) are shown in figure 1. Each differ in their general arrangement of lifting surface geometry and resulting aerodynamic characteristics.

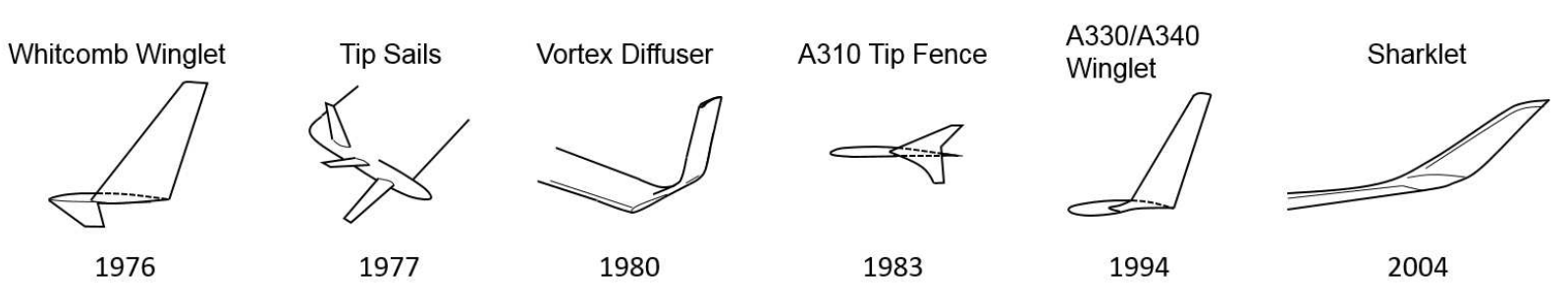

Figure 1. Non-planar wing tip device geometry and year of publication.

The first practical use of non-planer wing tips was achieved by Somerville ${ }^{13}$ in 1910, described as 'upcurled wing ends,' to enhance a biplane's lateral stability. Decades later, fundamental investigations into non-planar wing tip geometries consisted of simple end plates. ${ }^{14-16}$ These end plates were shown to increase the effective span of the wing. Whitcomb ${ }^{7}$ found that high aspect ratio end-plates were able to achieve greater gains in efficiency compared to a planar wing of equivalent span and lift; improving the lift-to-drag ratio by nearly a factor of two when compared to a tip extension with equivalent root bending moment.

Jones and Lasinski ${ }^{17}$ conducted a numerical study of the comparison of winglets and tip extensions with different weight constraints. It was concluded that the addition of a winglet did not provide a definite advan- 
tage over a tapered wing extension. $\mathrm{Kroo}^{18}$ also found that winglets provide no advantage over planar wings. Asai ${ }^{19}$ came to a similar conclusion stating that the reduction of induced drag achieved by winglets would be offset by the addition of wetted area (thus increasing parasitic drag) that did not contribute to lift. Thus, tip extensions were found to provide a slight improvement over winglets. However, efforts such as that by Heyson et al. ${ }^{20}$ conclude that for equivalent increases in the root bending moment, a winglet would provide a greater gain in induced efficiency than a tip extension. Computational studies conflict depending on: the drag models incorporated (some neglect viscous drag); how the bending moments are evaluated (structural constraints); the baseline geometry and associated flight envelope; the parametrised geometry; and level of flow solver fidelity.

More extreme examples of non-planar lifting surfaces are ring wings, ${ }^{21}$ box-wings ${ }^{22}$ and joined wings. ${ }^{23,24}$ Each of these are closed configurations with the aim of reducing the influence of wing tip vortices. ${ }^{25}$ The box wing configuration, among all non-planer configurations, has been shown to achieve the minimum possible induced drag for a given lift and height-to-span ratio. ${ }^{1} \mathrm{C}$-wings, which are very similar in geometric configuration to vortex diffusers,${ }^{9}$ have been considered a compromise between a box-wing and a winglet; theoretically providing a reduction in the induced drag that approaches that of the closed arrangement while additionally reducing the viscous drag penalty incurred by large wetted areas. ${ }^{25,26}$ Furthermore, the C-wing has been recognised to have the potential to replace the conventional horizontal stabiliser to provide pitch control. $^{25}$ The development of a systematic wing optimisation algorithm by Gage ${ }^{27}$ identified the C-wing configuration as an optimal solution while attempting to minimise the induced drag of a planform with fixed lift, span and height.

A numerical study by Slingerland and Verstraeten, ${ }^{28}$ with the inclusion of viscous drag and root bending moment for structural consideration, focused on the drag characteristics of optimally span-loaded planar wings, wings with winglets and C-wings. Planform geometry was fixed and the optimisation goal was to optimally span-load all lifting surfaces. It was found that optimally loaded non-planar wings had greater induced efficiency than elliptically loaded planar wings. They found that winglets only benefit wings with span constraints and also found no advantages to C-wing configurations.

Ning and $\mathrm{Kroo}^{29}$ highlighted that the lift coefficient at cruise condition of a given wing is often very different from the lift coefficient reached when undergoing a manoeuvre. They found that winglets performed better than tip extensions under high manoeuvre loads. Additionally they concluded that C-wings can achieve a slightly lower total drag compared to winglets for a constant positive pitching moment about the aerodynamic centre, especially for wings with span constraints enforced.

Jansen et al. ${ }^{30}$ solved a series of wing design optimisation problems using different types of drag models (starting with induced, then adding compressibility and viscous drag). They found in all cases that a non-planar wing configuration provided significant drag reduction from the reference planform with span constraints. A box-wing configuration closely followed by a C-wing configuration gave the greatest reductions in induced drag. When the wing span was not constrained the optimal solution was a raked wing tip extension.

Lack of consensus for what defines a truly optimal wing configuration motivates the need for further work in this area. Previous efforts largely treat aerodynamic optimisation of wing planforms as a uni-model mathematical problem; there remains a lack of optimisation that appropriately investigate multi-objective optimisation. As a result, many efforts can be deemed, in many ways, as artificially driving the solution. Additionally, aerodynamic optimisations can be poorly parametrised and very rigid, guaranteeing sub-optimal results.

The first aim of this investigation is to develop a sufficiently accurate flow solver to enable the optimisation process with accurate computation of aerodynamic moments and forces while remaining computationally inexpensive. Inaccurate modelling will lead to pseudo-optimal designs. Steps have been taken to allow more accurate evaluation of the induced drag while viscous drag is also taken into consideration.

Secondly, the wing optimisation process is to be given as much flexibility as possible. This has been accomplished by representing a series of wing segments with variable sweep, span, taper, twist, dihedral, aerofoil 
section, and aerofoil orientation (to allow local circulation to be either positive or negative) to compose the wing. Further variables include root chord, angle of attack and centre of gravity location. Steps have also been taken to allow for a wide scope of variable parametrisation. The optimiser is capable of adding or removing additional wing segments made possible by the use of a structured genetic algorithm (sGA) with embedded constraint based reasoning capability to efficiently guide solutions in the design space. Finally, the affects of pitch stability on the aerodynamic design optimisation of the wing configuration are explored.

\section{Model Description}

The difficulty in aerodynamic optimisation is being able to define an analysis method that is sufficiently simple to operate thousands of times within a parametrised search space, but is sophisticated enough to capture enough consideration that determines a local geometry that feeds into a globally optimal system. ${ }^{31}$

Aerodynamic optimisation using Computational Fluid Dynamics (CFD) is a challenging problem to approach for four main reasons: 1) The objective function distributions can be extremely rough, as pointed out by Obayashi and Tsukahara, ${ }^{32}$ originating from the non-linearity of governing flow equations; 2 ) The design space is highly multi-dimensional; 3) Grid meshing problems between function evaluations of different geometry; and 4) Single Function evaluations are very expensive. For example, a single aerodynamic evaluation using a Navier-Stokes calculation can take several hours and can even require extreme computational resource. ${ }^{33,34}$ One of the key challenges is the construction of a new computational grid for each evaluation. Novel complex geometries are generally constructed manually which is very time consuming. ${ }^{35}$ In order to overcome this automatic mesh constructions are required. CFD ultimately restricts the allowable design space either through computational needs or through how the baseline geometry is allowed to deform with a fixed mesh. ${ }^{36}$

Alternatively, lower fidelity flow solvers (often regarded as 'first-order' solvers) such as aerodynamic panel methods can be used to analyse arbitrary wing configurations. The flow solver has been integrated into a gradient-free optimiser due to the scope and number variables used, and can independently activate and deactivate certain variables. No a priori knowledge of the design space is known and so, navigation of discontinuities within the design space and the existence of multiple local-optima require the application of a gradient-free optimisation architecture.

\section{A. Aerodynamic Model}

The aerodynamic forces and moments are evaluated by a potential flow solver governed by Laplace's equation. ${ }^{37}$ The lifting surface geometry is automatically discretised in both span-wise (NP) and chord-wise (NC) panels into vortex rings based on a user defined fidelity over the camber line of some aerofoil.

A drag-free wake is shed by trailing vortex rings and extends 30 wing semi-spans aft of the surface with the Trefftz plane located 15 semi-spans. ${ }^{38}$ Induced drag is calculated using pairs of survey points at the Trefftz plane to compute relevant velocities and the potential jump across the wake. ${ }^{38,39}$ The induced drag is then approximated by summing over the wake panels:

$$
D_{\text {ind }}=-\frac{1}{2} \rho \sum_{i=1}^{N P} \Delta \varphi_{i} \frac{\partial \varphi}{\partial \mathbf{n}_{i}} \Delta y_{i}
$$

In consideration of the total planform drag, one must take into consideration the viscous drag. Viscous drag will comprise of a significant fraction of the total drag budget and so cannot be neglected. The main effect to the optimisation process when considering viscous drag is that this will add a penalty to wetted areas incurred, particularly from surfaces that do not contribute to lift: such as winglets. Hence, consideration of the viscous drag is particularly important as the optimisation architecture will allow the wing planform wetted area to vary. Fixing the wetted area does not lead to an effective optimisation of a fair comparison of relative performance between planar and non-planar wings.

Several approaches are available to extend the potential aerodynamic model to include viscous boundary layer effects, such as coupling the potential flow solver with a boundary layer solver. ${ }^{37}$ A computationally simpler method is to construct a database of relevant two-dimensional aerofoil data over the range of sectional Reynolds numbers to be expected. This enables the use of sectional aerofoil drag polars to relate sectional 
lift coefficients to the sectional drag coefficient as a function of sectional Reynolds number. ${ }^{28-30}$ This enables the assumption that the viscous drag coefficient of the aerofoil varies quadratically with the section lift coefficient:

$$
c_{d_{p}}=c_{d_{p 2}}(R e) c_{l}^{2}+c_{d_{p 1}}(R e) c_{l}+c_{d_{0}}(R e)
$$

The viscous drag model uses a range of drag polars for different aerofoil sections over the Reynolds number range of $2 \times 10^{6}$ to $10 \times 10^{6}$. The three coefficients $\left(c_{d_{p 2}}, c_{d_{p 1}}, c_{d_{p 0}}\right)$ are determined by interpolating between drag polars at different sectional Reynolds numbers and lift coefficients.

The vortex ring method developed with the embedded two-dimensional aerofoil drag-polar data has been validated against experimental wind tunnel data for a planar wing collected by Sivells. ${ }^{40}$ The experimental and calculated lift-curve-slope and drag polar are shown in figure 2. Different spatial discretisation of the aerody-

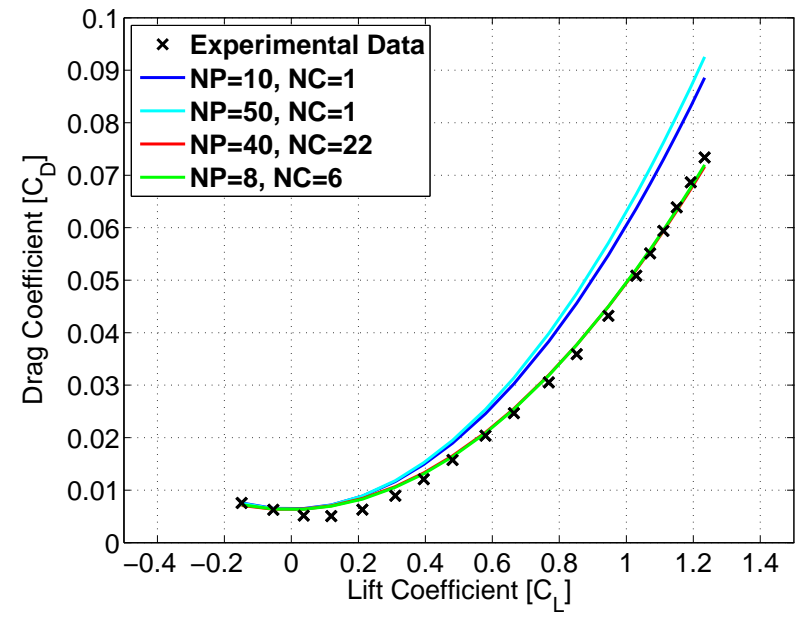

(a) Experimental and calculated lift-curve-slope.

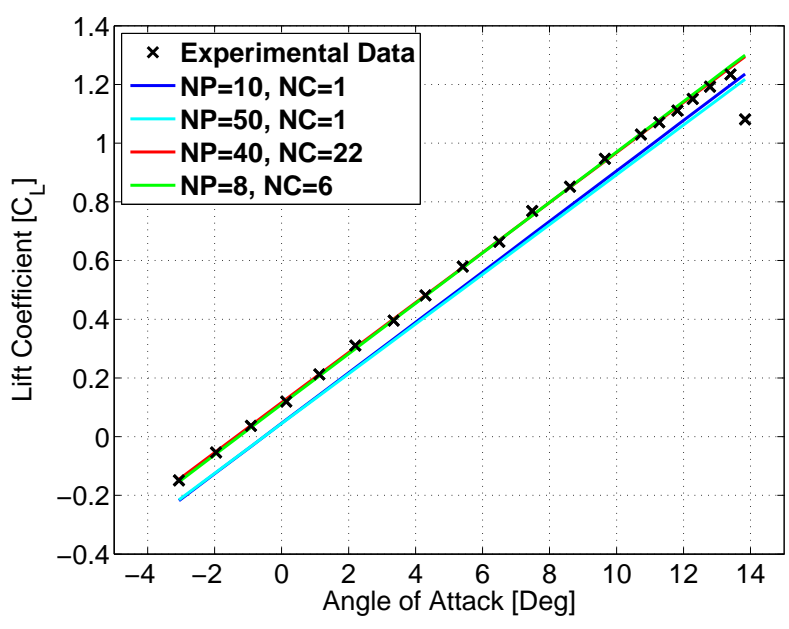

(b) Experimental and calculated drag polar.

Figure 2. Comparison of experimental and calculated data for a wing using different span-wise (NP) and chord-wise (NC) aerodynamic panel discretisation.

namic panelling over wing the semi-span has been investigated in-order to optimise the balance of accuracy and computational time. Panelling distributed in the span-wise direction alone has been deemed unsuitable for the accurate prediction of induced drag, although it is sufficient for the evaluation of the wing lift. This is evident from figure 2(a) where increasing the span-wise panels only from $\mathrm{NP}=10$ to $\mathrm{NP}=50$ (with only a single chord panel, $\mathrm{NC}=1$ ) slightly improves the evaluation of the lift coefficient but further deteriorates the drag coefficient evaluation. A fundamental statement within thin aerofoil theory is that the camber line is considered a stream-line of the flow over which the vortex strength varies. The nature of the panel method is devised to conceptually obtain values for vortex strength which make the modelled wing a streamline of the flow and also satisfy the Kutta condition. Increasing span-wise panels serves to better resolve vortex strength per unit length across the span (more precisely evaluating lift), but it does not sufficiently resolve the induced velocities (i.e. down-wash). Hence, increasing the number of chord-wise panels serves to distribute more control points along the camber line at which boundary conditions are applied; that is, at each control point the normal component of the velocity is zero. This velocity is the superposition of the uniform flow velocity and the velocity induced by the vortex panels. As a result, the induced velocity distribution over the wing is more accurately modelled (leading to a more accurate computation of the induced drag), and the Kutta condition is applied more precisely. Chord-wise panels are also necessary to resolve the effects of geometric twist.

40 span and 22 chord panels were found to give the greatest accuracy relative to the experimental data, giving a root-mean-squared error of $0.824 \%$ and a total evaluation time of approximately 10 minutes. For the optimisation process the spatial resolution of aerodynamic panels has been set at 8 span and 6 chord panels. Reducing the span and chord panelling served to bring a single flow evaluation to under half a second ( 0.47 seconds) while maintaining sufficient accuracy: the root-mean-squared error was evaluated to be $1.22 \%$. Within the optimisation process aerodynamic panels (vortex rings) are distributed proportionately over a given wing to provide, as closely as possible, a consistent span-wise discretisation between changing 
arbitrary wings. Motivation for this is that results obtained for a given body are sensitive to the spatial resolution of the panels used. ${ }^{41}$ Thus, while chord-wise panelling is fixed, the span-wise panelling can adapt between arbitrary wings in order to maintain equivalent span-wise spatial resolution.

At low lift coefficients, the panel method over-predicts the total drag by up to $14.08 \%$ as reflected in figure 2(a). At low lift coefficients the viscous drag model dominates. The simplifying assumption of quadratic variance of viscous drag with sectional lift coefficient does not give consistent accuracy here. Figure 2(b) additionally illustrates limitations that exist at high angles of attack: flow separation is not modelled by the panel method.

\section{B. Optimiser: Structured Genetic Algorithm}

The field of optimisation is expansive, and the choice of suitable optimisation architecture is highly problem dependant. ${ }^{42}$ Considerations must be made with regards to: the types of design variables (e.g. discrete and/or continuous); the number of constraints; the number of variables; and the properties of the design space (i.e. presence of local optima and discontinuities). Genetic algorithms (GAs) search for optimal solutions using multiple candidate solutions within a design space simultaneously and stochastically. GA is an adaptive search technique that is inspired by both the natural inheritance of genes, from parents to offspring, and the Darwinian theory of natural selection; a primary aspect of evolution. ${ }^{43}$ GAs have been shown capable for preventing a search from getting stuck in locally optimal solutions, and are capable of identifying a range of different viable solutions and also serve to identity different disciplinary compromises among design objectives. ${ }^{27,33,34}$

A structured genetic algorithm ${ }^{44}$ (sGA) has been developed for aerodynamic optimisation. The basic framework of the sGA is based on the NSGA-II ${ }^{45}$ however there are significant differences. In NSGA-II infeasible solutions which violate one or more constraints are considered completely inferior to all other solutions. Infeasible solutions, even those with superior objective function evaluations, are not used at all in searching for Pareto-optimality; even if a single constraint violation is very small. To counter this, constraint based reasoning is enforced directly through proportional penalty functions which enables the existence of both feasible and infeasible solutions within the population. Reasoning for this is motivated by the assumption that a Pareto-optimal set of solutions may exist between a region of feasibility and infeasibility. ${ }^{46}$

The sGA, by design, allows candidate solutions to have a dynamic structure in the genotype chromosome. This enables different (or alternative) types of solutions to co-exist in the same population, and also works as a distributed memory within the population which propagates from generation to generation. ${ }^{44}$ It is capable of preserving multiple schemata in the genotype of solutions. This is achieved through a gene activation mechanism embedded within the genotype search space to effectively turn on (active) and turn off (passive) different design variables. The active and passive genes mimic dominant and recessive gene structures in genetics. ${ }^{44}$ A given solution will have a set of 'high level' genes which can activate or deactivate sub-sets of the genotype description. This high level gene organisation in turn governs the genotype-phenotype mapping dynamically expressing if the property will be active and so present phenotypically. This allows all chromosomes to be of equal length with all variables present, but the 'active' chromosome can change length.

\section{Design Variables}

An individual wing within the allowable design space can be represented by one to four trapezoidal wing segments, whereby individual segments and associated design variables can be activated or deactivated by the structured genetic algorithm. The geometry of each segment is described by six design variables: sweep, span, taper, twist, dihedral, and aerofoil section. The wing tip segment is given a seventh variable for aerofoil orientation (to allow local circulation to be either positive or negative). Further variables include root chord, angle of attack and centre of gravity location: a total of 28 accessible design variables. The sGA cannot deactivate the root wing segment and, a prerequisite for activation is that the panel before is already, or is simultaneously, made active: i.e. panel four cannot be activated unless both panels two and three are active. Wing segments must form a continuous structure in which the tip chord of each segment is the same as the 
root chord for the next segment.

The sGA will allow the addition and removal of new wing segments during the optimisation process. The parametrisation boundaries (maximum and minimum) for each variable have been kept large as the sGA is capable of quickly identifying useful sub-ranges of variables.

\section{Optimisation Constraints}

Owing to limitations of the panel method developed, steps must be taken to ensure that the wing segments do not arrange themselves into configurations that the flow solver cannot appropriately analyse: for instance, if two wing segments penetrate one another or if relative dihedral angles of neighbouring vortex ring panels approach 180 degrees (panels fold on top of each other). These types of structure often lead to a vortex line intersecting or moving too close to a control point forcing the aerodynamic influence matrix to become ill-conditioned. The vortex ring method gives a set of linear algebraic equations represented by the flow boundary conditions at the control point of each panel. Hence, if a control point is intersected or is in too close proximity to a vortex line the linear system of algebraic equations no longer has a unique solution due to this intersection.

To remove the possibility of such configurations basic geometric constraint checks are made on planform geometry ahead of the aerodynamic analysis with a design constraint, $\Gamma_{\text {limit }}$, actively limiting the relative angle between neighbouring panels. If these critical geometric constraint checks are violated then the wing cannot be analysed and will be rejected by the algorithm ahead of analysis. All other constraint checks are made post-analysis. Solutions that fail to meet constraints at this stage are penalised through proportional penalty functions applied to the objective functions. This allows both infeasible and feasible solutions to co-exist in the population but forms a population structured hierarchy that enables the sGA to guide the optimisation. This concept is deemed particularly valuable as solutions with superior objective functions may violate a constraint by a very small amount (e.g. maximum span constraint violated by $1 \%$ ). Thus instead of simply rejecting the solution configuration based on the fact that it failed one or more constraint checks, and losing an individual with favourable traits, the solution is allowed to remain within the population but with a proportionately offset objective function. This means that large or multiple constraint violations will force a solution to rank as less superior within the population, while solutions with small constraint violations are recognised as more superior (but never most superior) and remain in the population to evolve.

Additional constraints that can be enforced in the optimisation process are minimum lift requirements, maximum root bending moment and a static margin constraint based on the centre of gravity longitudinal location. Each also applies penalties to candidate solutions through proportional penalty functions. 


\section{Wing Extension Optimisation: Retrofit}

The baseline geometry and flow conditions are taken to be the same as the planform with a NACA 65-210 aerofoil used by Sivells. ${ }^{40}$ The aim is to identify a range of simple wing extension geometries for a fixed planform. Both span constrained and span unconstrained optimisation runs that result in a decrease of induced drag while minimising the increase of parasitic drag are considered. Root bending moments, changes in wetted area, and changes in span loading have also been monitored. The geometry for both the wing section and the vortex wing model arrangement is seen in figure 3, and planform parameters are summarised in table 1.

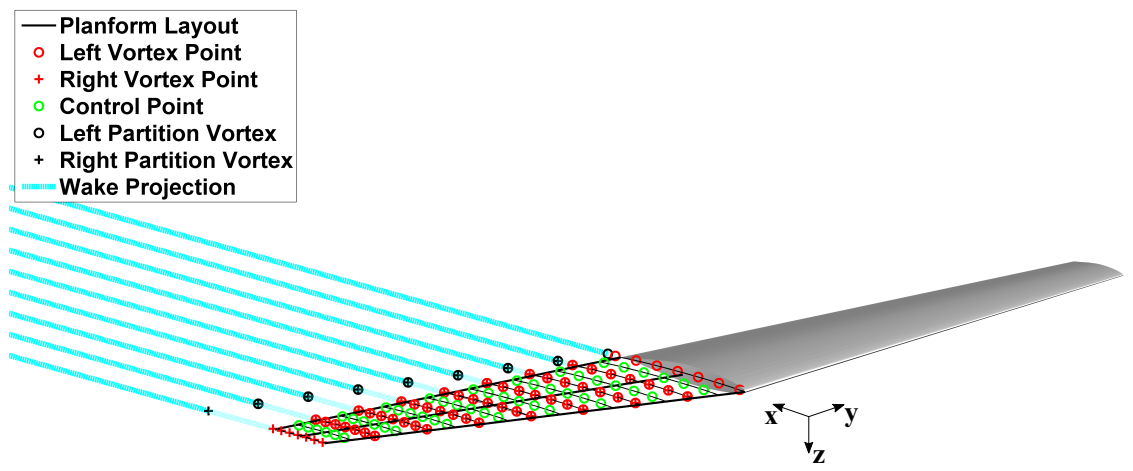

Figure 3. Baseline geometry used by Sivells ${ }^{40}$ represented by port wing. Starboard wing represents the vortex panel method used to model the wing with 8 span-wise panels and 6 chord-wise panels. Freestream velocity taken in positive $\mathrm{x}$-direction.

\begin{tabular}{|c|c|}
\hline$\Lambda$ & $-2.726^{\circ}$ \\
\hline $\mathrm{b}$ & $2.286 \mathrm{~m}$ \\
\hline$C_{\text {root }}$ & $0.726 \mathrm{~m}$ \\
\hline$\lambda$ & 0.400 \\
\hline $\mathrm{AR}$ & 4.500 \\
\hline
\end{tabular}

Table 1. Baseline wing description.

Only a small number of variables are needed as only the wing tip extension geometry is optimised and the planform remains fixed. The extension is modelled using one trapezoidal wing segment with variables of segment span, taper ratio, sweep, dihedral and planform angle of attack. If optimal the sGA can completely remove the wing extension. A NACA 65-210 aerofoil is used so that the extension would easily blend with the existing geometry. The root bending moment constraint helps to encourage lower tip loading of the wing while also limiting span extension for unconstrained span optimisation. The optimisation is presented as follows:

minimise: $D_{\text {ind }}$ and $D_{\text {para }}$

With respect to variables $\left\{\begin{array}{ll}0 \leq \Lambda_{i} \leq 45 & {[\mathrm{deg}]} \\ 0.1 \leq \lambda_{i} \leq 1 & \\ 0 \leq \Gamma_{i} \leq 135 & {[\mathrm{deg}]} \\ 0 \leq b_{i} \leq 2 & {[\mathrm{~m}]} \\ 0 \leq \alpha_{i} \leq 12 & {[\mathrm{deg}]}\end{array} \quad\right.$ Subject to $\left\{\begin{array}{l}1-\frac{L}{L_{\text {ref }}} \leq 0 \\ \frac{b_{\text {ref }}}{h_{\text {wingtip }}}=0.25 \\ \Gamma_{\text {limit }}=45 \quad[\text { deg }] \\ M_{\text {root }} \leq m_{\text {limit }} M_{\text {root }} \\ \frac{b}{b_{\text {ref }}} \leq \text { slimit }_{\text {limef }} b_{\text {ref }}\end{array}\right.$

The scaling coefficients $m_{\text {limit }}$ and $s_{\text {limit }}$ are used to define the maximum allowable root bending moments (RBM) and wing span constraints respectively. The sGA ran for 300 generations with a population of 50; deemed to be sufficient based on convergence studies with the algorithm. Figure 4 shows four Pareto fronts: 1) Span constrained with no RBM constraint $\left(m_{\text {limit }} \rightarrow \infty, s_{\text {limit }}=1\right)$; 2$)$ RBM constraint 1 with no 
span constraint $\left.\left(m_{\text {limit }}=1.22, s_{\text {limit }} \rightarrow \infty\right) ; 3\right)$ RBM constraint 2 with no span constraint $\left(m_{\text {limit }}=\right.$ 1.36, $\left.s_{\text {limit }} \rightarrow \infty\right)$; and 4) RBM constraint 3 with no span constraint $\left(m_{\text {limit }}=1.52, s_{\text {limit }} \rightarrow \infty\right)$. From the Pareto optimal solutions some selected wing geometries are shown in figure 5.

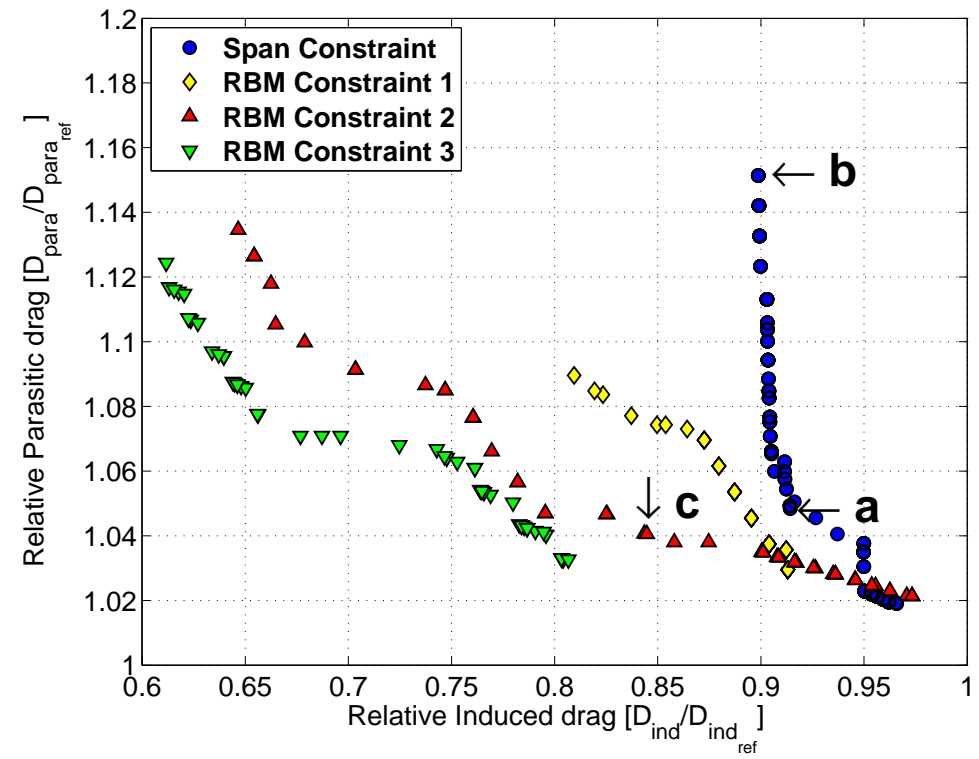

Figure 4. Pareto fronts of optimised wing extensions: Span constraint $\left(m_{\text {limit }} \rightarrow \infty, s_{\text {limit }}=1\right)$; RBM constraint $\mathbf{1}\left(m_{\text {limit }}=1.22, s_{\text {limit }} \rightarrow \infty\right)$; $\mathbf{R B M}$ constraint $\mathbf{2}\left(m_{\text {limit }}=1.36, s_{\text {limit }} \rightarrow \infty\right)$; and $\mathbf{R B M}$ constraint $\mathbf{3}\left(m_{\text {limit }}=\right.$ $\left.1.52, s_{\text {limit }} \rightarrow \infty\right)$.

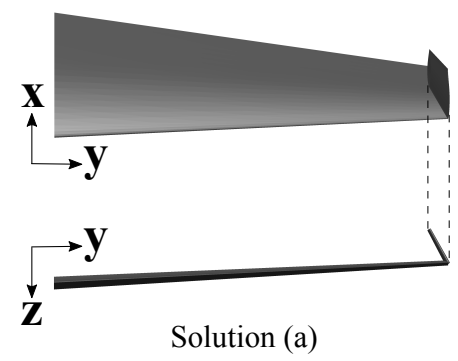

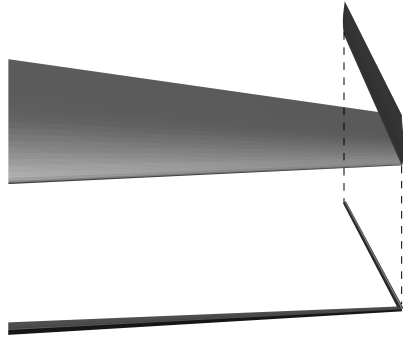

Solution (b)

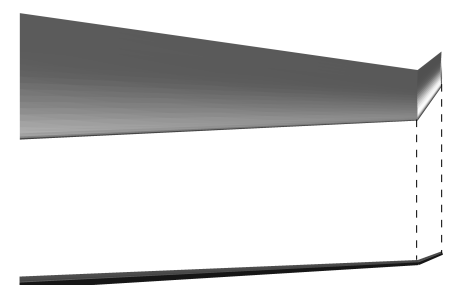

Solution (c)

Figure 5. Physical representation of solutions identified along Pareto fronts in figure 4.

The Pareto front representing the optimisation of the extension with span constraint of the base geometry $\left(s_{\text {limit }}=1\right.$, resulting in wing extensions with dihedral $\geq 90^{\circ}$ ) exhibits asymptotic behaviour at

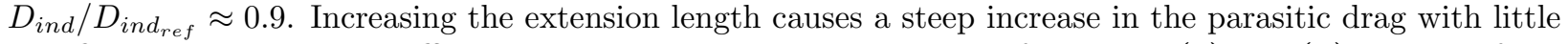
benefit gain in the induced efficiency. The geometric representations of solutions (a) and (b) over this front are shown in figure 5 where the physical increase in the wetted area is obvious. Wing solution (a) is the optimal solution in this front decreasing the total drag by $2.4 \%$. Wing (b) has the largest extension acting to increase the total drag by $1.34 \%$. Increases in induced efficiency achieved by solution (b) are negated by the drag penalties incurred from parasitic drag due to increased wetted area.

Wing solutions with span constrained only by the root bending moment are seen to offer greater gains in induced efficiency, up to $40 \%$, however result in greatly increased root bending moments as the extension span increases, dihedral lowers, and sweep increases. Wing solution (c), highlighted in figure 4 and geometric representation shown in figure 5, achieved a greater reduction in the total drag of $9.1 \%$ relative to solution (a) for an equivalent root bending moment increase. Figure 6 shows the relative effects of each of the extension configurations on the span loading over the wing.

As the RBM constraint increased, more optimal solutions with greater total drag reduction were found 


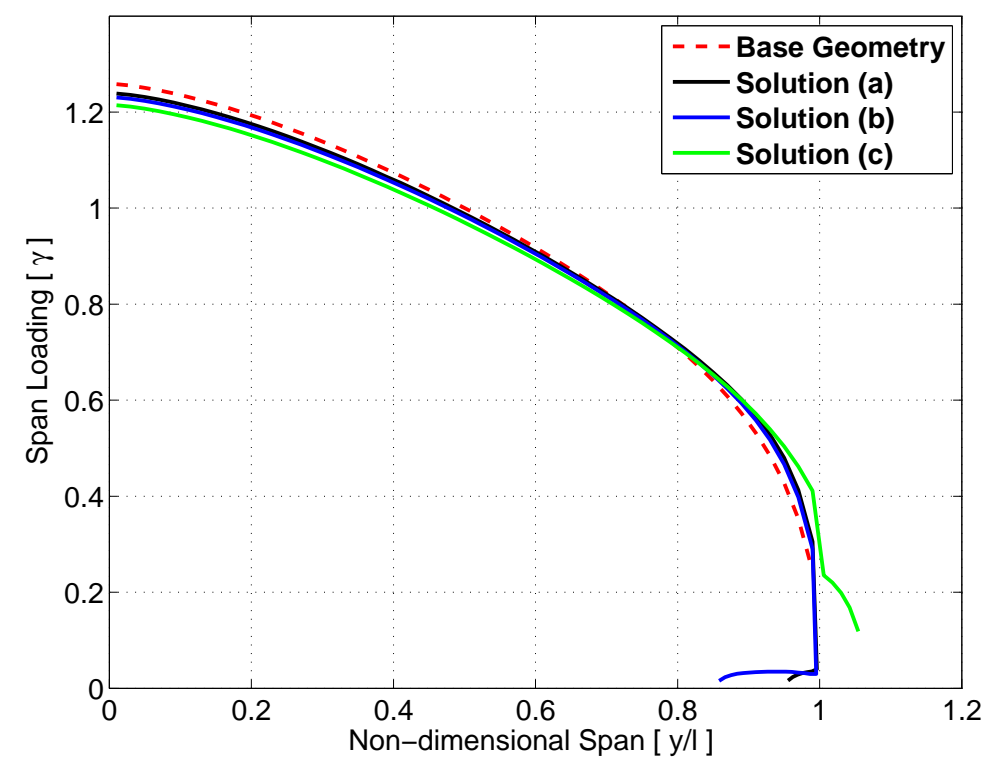

Figure 6. Span loading distributions for wing geometries shown in figure 5 .

with increased length/sweep of the extension and lowering the dihedral angle. Solutions defined by RBM constraint 3 (largest allowable RBM) were found to have lower wing tip dihedral than those solutions defined by RBM constraint 1 (smallest allowable RBM). Each front identified share a similar shape with a visible plateau of the parasitic drag increase. The physical geometry of the wing extensions in these regions were found to be similar with small differences in the tip dihedral angle and sweep: demonstrating the sensitivity of the induced drag to the wing tip geometry.

\section{Integrated Planform Wing tip and Winglet/Tip-extension Optimisation}

The wing design problem is examined in which the outer $25 \%$ of the baseline geometry (regarded as the planform wing tip) is optimised along side the end-plate. Two trapezoidal wing sections are optimised with the following design variables: span, taper ratio, dihedral, sweep, local twist and aerofoil section for each section, and planform angle of attack. The selected aerofoil sections allow for readjustment of the camber between wing sections: NACA 65-210 $\left(A_{\text {ero } 1}\right)$ or NACA $0010\left(A_{\text {ero } 2}\right)$ may be selected. Options between aerofoils require additional parasitic drag models as this is based on two-dimensional aerofoil drag polars. The sGA can activate or deactivate the two segments, while $75 \%$ of the planform remains fixed. The optimisation is presented as follows:

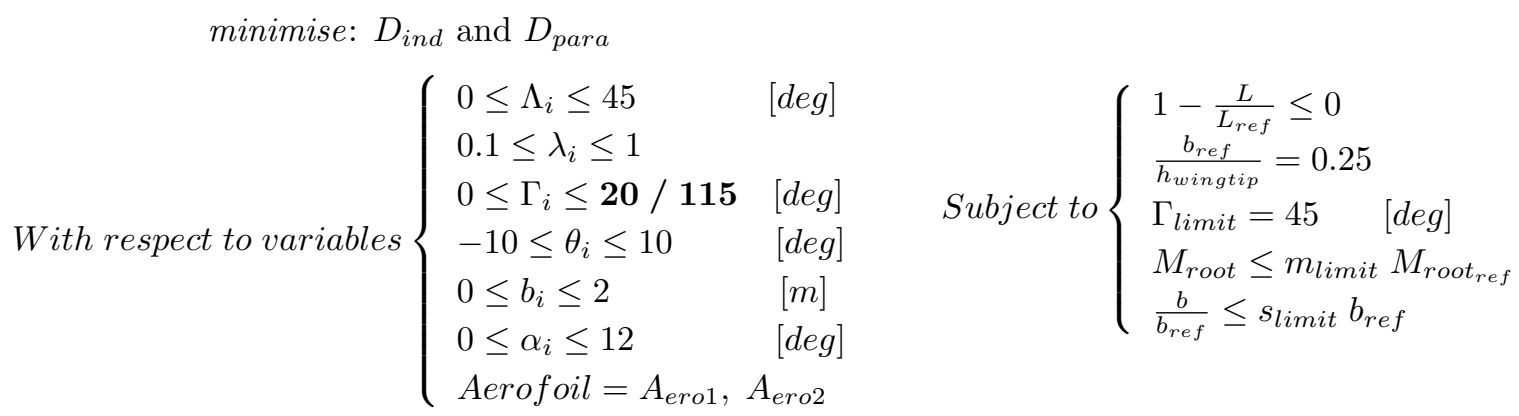

The sGA executed 500 generations with a population of 100; deemed to be sufficient based on convergence studies with the algorithm. Note that there are two parametrised ranges for sweep $(\Gamma)$; this has been done to separate winglets and wing tip extension solutions to assess relative performance in the parametrised design space. Hence winglets are considered to be extensions of the wing with a dihedral angle greater than $20^{\circ}$, while tip extensions have a dihedral angle lower than $20^{\circ}$. 


\section{Integrated Wing tip and Winglet Optimisation}

The resulting Pareto fronts for integrated wing tip/winglet optimisation are shown in figure 7: 1) Span constrained with no RBM constraint $\left(m_{\text {limit }} \rightarrow \infty, s_{\text {limit }}=1\right)$; 2$)$ RBM constraint 1 with no span constraint $\left.\left(m_{\text {limit }}=1.22, s_{\text {limit }} \rightarrow \infty\right) ; 3\right) \mathrm{RBM}$ constraint 2 with no span constraint $\left(m_{\text {limit }}=1.36, s_{\text {limit }} \rightarrow \infty\right)$; 4) RBM constraint 3 with no span constraint $\left(m_{\text {limit }}=1.52\right.$, $\left.s_{\text {limit }} \rightarrow \infty\right)$; and 5$)$ RBM constraint 3 with span constraint $\left(m_{\text {limit }}=1.52, s_{\text {limit }}=1.10\right)$. The optimisations executed with no span constraint limit the maximum allowable wing span through the trade-off between the increase in the root bending moment due to a reduction in induced drag. In figure 7 wing solutions (a), (b), (c) and (d) have been selected to show how the wing tip/winglet geometry changes over the defined Pareto optimal solution range. The physical representations of these wings are shown in figure 8 with the resulting span loading distributions shown in figure 9.

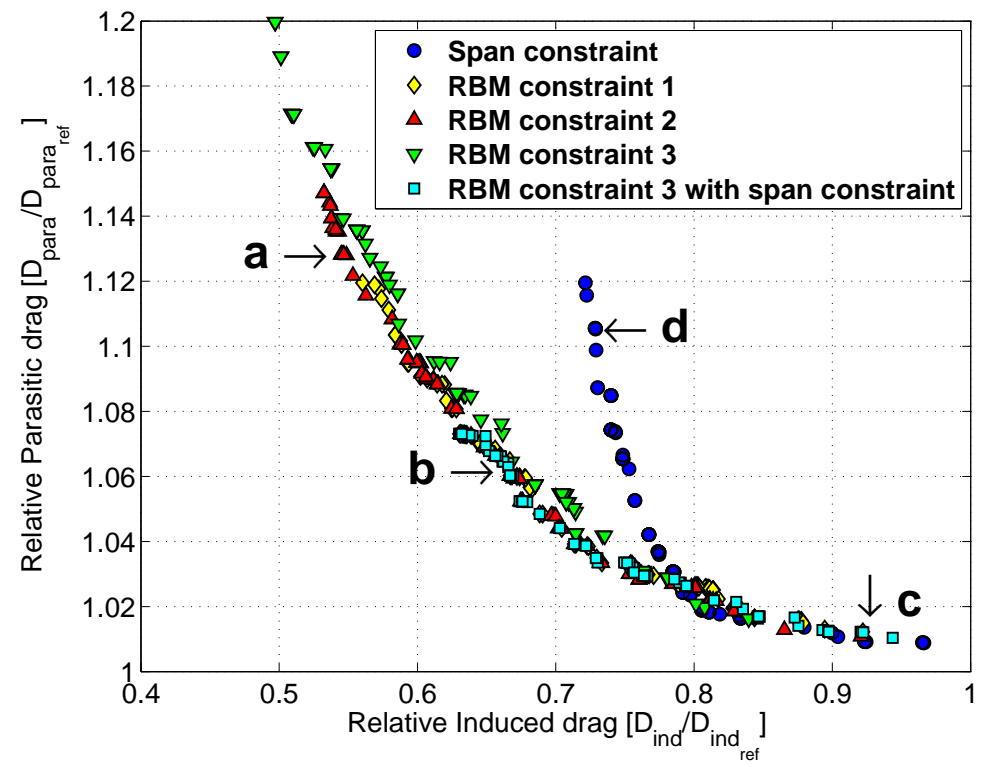

Figure 7. Pareto fronts of optimised wing tip/winglet: Span constraint $\left(m_{\text {limit }} \rightarrow \infty, s_{\text {limit }}=1\right)$; RBM constraint $1\left(m_{\text {limit }}=1.22, s_{\text {limit }} \rightarrow \infty\right)$; RBM constraint $\mathbf{2}\left(m_{\text {limit }}=1.36, s_{\text {limit }} \rightarrow \infty\right)$; $\mathbf{R B M}$ constraint $\mathbf{3}\left(m_{\text {limit }}=\right.$ $\left.1.52, s_{\text {limit }} \rightarrow \infty\right)$; and RBM constraint 3 with span constraint $\left(m_{\text {limit }}=1.52, s_{\text {limit }}=1.10\right)$.

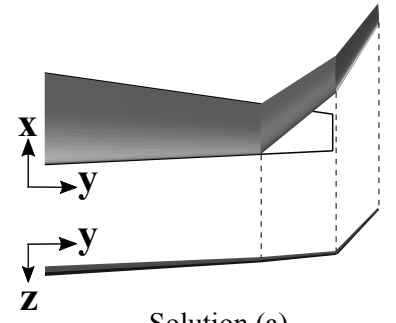

Solution (a)

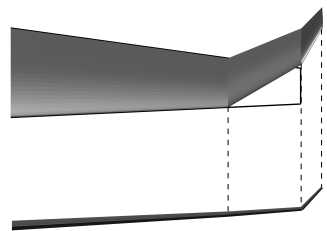

Solution (b)

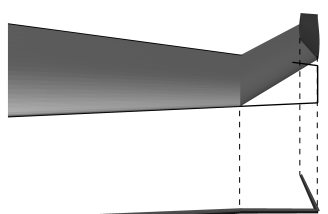

Solution (c)

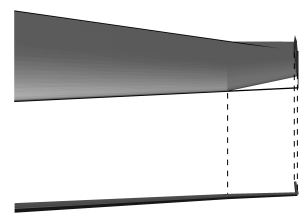

Solution (d)

Figure 8. Optimised wing tip/winglet configurations from Pareto fronts in figure 7. Baseline wing geometry is outlined.

The solutions shown in figure 7 coalesce into the same front within the allowable design space but identify limiting design boundaries. Solutions found at the lower extreme of the Pareto fronts around solution (c) reduced the total drag by up to $3.7 \%$ while having negligible influence on the RBM. The span constrained $\left(s_{\text {limit }}=1\right)$ Pareto shows an asymptotic increase in parasitic drag. This occurs due to the winglet's continued span extension as incremental improvement to the induced efficiency is made. Solution (d), with a total drag reduction of $10.8 \%$, restricted by span only is found on the cusp of solutions that begin to increase 
total drag through significant gains in parasitic drag.

Moving along the main Pareto from solution (c) to (a) the winglet is seen to lower its dihedral angle and increase sweep. The wing tip is also seen to increase in sweep. The most superior solution, solution (a) with a total drag reduction of $19.3 \%$ for a $9.4 \%$ span extension and a $22.5 \%$ increase in RBM, shows an increase in dihedral and sweep over the wing span with a continuous decrease in local chord and a continuous variation of local twist. The way in which solutions vary over the front can be seen in figure 8. Solution geometry from wing (c) to wing (b) and then wing (a) show the trends described over the front.

The modified geometry results in changes in span loading at the wing tip with effects shown in figure 9 . The highly swept outboard sections of wing solutions (a), (b), and (d) cause a sudden drop in span loading. This reflects a limitation of the first order VRM solver developed, arising from the large and instantaneous increase in sweep angle. This causes neighbouring aerodynamic panels to experience very different relative velocities leading to a discontinuity in the lift distribution. In reality, this drop in loading would not be as steep meaning that the RBM calculated is expected to be under predicted.

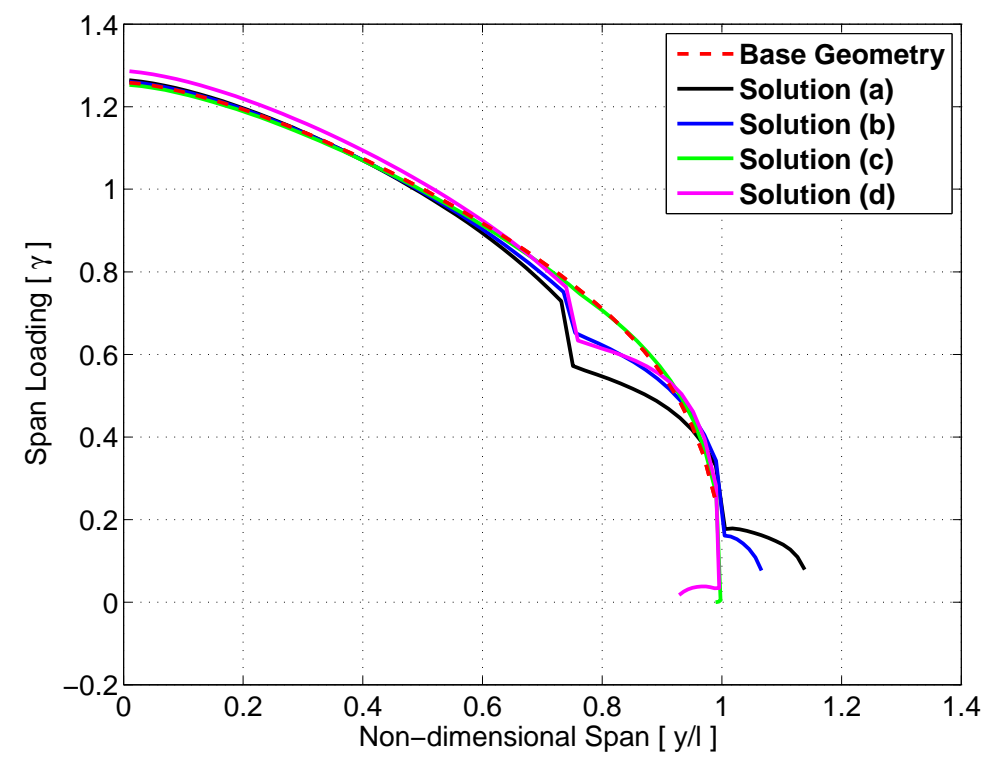

Figure 9. Span loading distributions for wing geometries shown in figure 8 .

\section{Integrated Wing tip and Tip Extension Optimisation}

The resulting Pareto fronts for integrated wing tip/tip extension optimisation are shown in figure 10: 1) RBM constraint 1 with no span constraint $\left(m_{\text {limit }}=1.22\right.$, slimit $\left.\rightarrow \infty\right)$; 2$)$ RBM constraint 2 with no span constraint $\left(m_{\text {limit }}=1.36, s_{\text {limit }} \rightarrow \infty\right)$; 3$)$ RBM constraint 3 with no span constraint $\left(m_{\text {limit }}=\right.$ $\left.1.52, s_{\text {limit }} \rightarrow \infty\right)$; and 4$)$ RBM constraint 3 with span constraint $\left(m_{\text {limit }}=1.52, s_{\text {limit }}=1.10\right)$. Solutions (a), (b), and (c) are identified to demonstrate how the solutions vary across the front. The geometries of these solutions are given in figure 11; span loading distributions are shown in figure 12. 


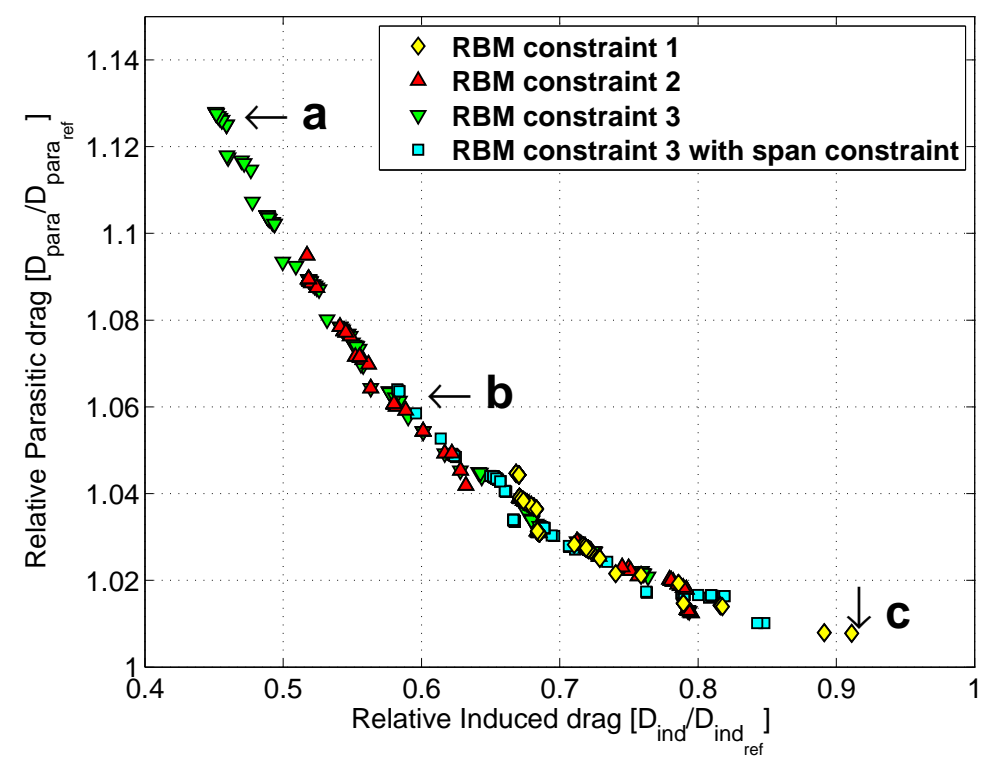

Figure 10. Pareto fronts of optimised wing tip/tip-extension: RBM constraint 1 ( $\left.m_{\text {limit }}=1.22, s_{\text {limit }} \rightarrow \infty\right)$; $\mathbf{R B M}$ constraint $\mathbf{2}\left(m_{\text {limit }}=1.36, s_{\text {limit }} \rightarrow \infty\right)$; RBM constraint $\mathbf{3}\left(m_{\text {limit }}=1.52, s_{\text {limit }} \rightarrow \infty\right)$; and RBM constraint 3 with span constraint $\left(m_{\text {limit }}=1.52, s_{\text {limit }}=1.10\right)$.

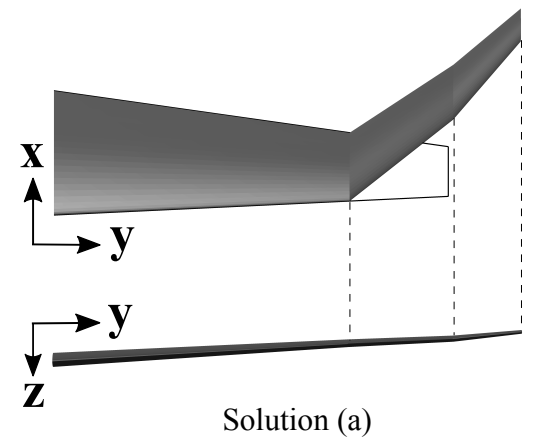

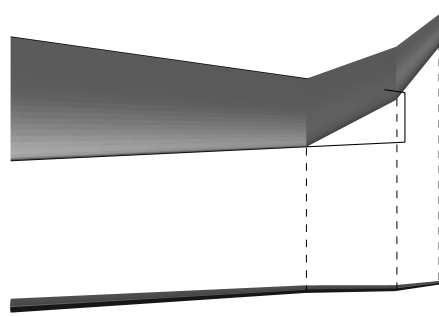

Solution (b)

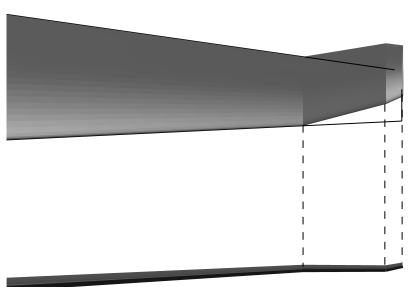

Solution (c)

Figure 11. Optimised wing tip/winglet configurations from Pareto fronts in figure 10. Baseline geometry outline shown.

The Paretos shown in figure 10 coalesce over the same front within the allowable design space. Design boundaries along the front are visible where one set of solutions end (limited by the optimiser constraints) but another set continues to follow the front (as defined by a larger RBM constraint for example). The fronts are not as steep as those seen in figure 7 for the winglet design; similar gains in induced drag can be made for less parasitic drag penalties incurred. Winglets are found to have a stronger influence on the parasitic drag as their span is not as robustly suppressed by the RBM constraints. As the Reynolds number of a wing extension is lower than the wing there will be a larger influence of the parasitic drag. Extensions of the wing, with low dihedral angles, are more sensitively limited by the design RBM constraint minimising the Reynolds number effects on the parasitic drag. Moving across the Pareto front (from solution (c) to solution (a)) the trend in wing geometry shown over the solution range is similar to that exhibited by the integrated wing tip/winglet optimisation: wing tip/tip extension sweep increases, and the tip extension increases in span. This is reflected in figure 11. Solution (a) is the optimal solution with a total drag reduction of $21.8 \%$ but suffers from a greatly increased RBM of over $50 \%$. Solution (b), optimised with both a RBM constraint and a span constraint reduced the total drag by $19.8 \%$ with an increased span of $9.1 \%$ and a RBM increase of $28.7 \%$.

Comparing the Pareto optimal results obtained for integrated wing tip/tip extension optimisation from figure 7 solution (a) and figure 10 (b), for equivalent reduction in the total drag the winglet was found to have a lower RBM for similar increase in wingspan. The winglet obtained higher drag penalties due to 
increased wetted area but also had a higher induced efficiency.

The span loading of wing solutions (a), (b), and (c) are given in figure 12. The discontinuous drop in loading due to flow solver limitations caused by an instantaneous change in sweep is seen. The tip extensions are more highly loaded relative to winglet solutions due to the lower dihedral angle. The inboard wing loading is seen to slightly reduce while increased tip loading is significant relative to the baseline wing span loading.

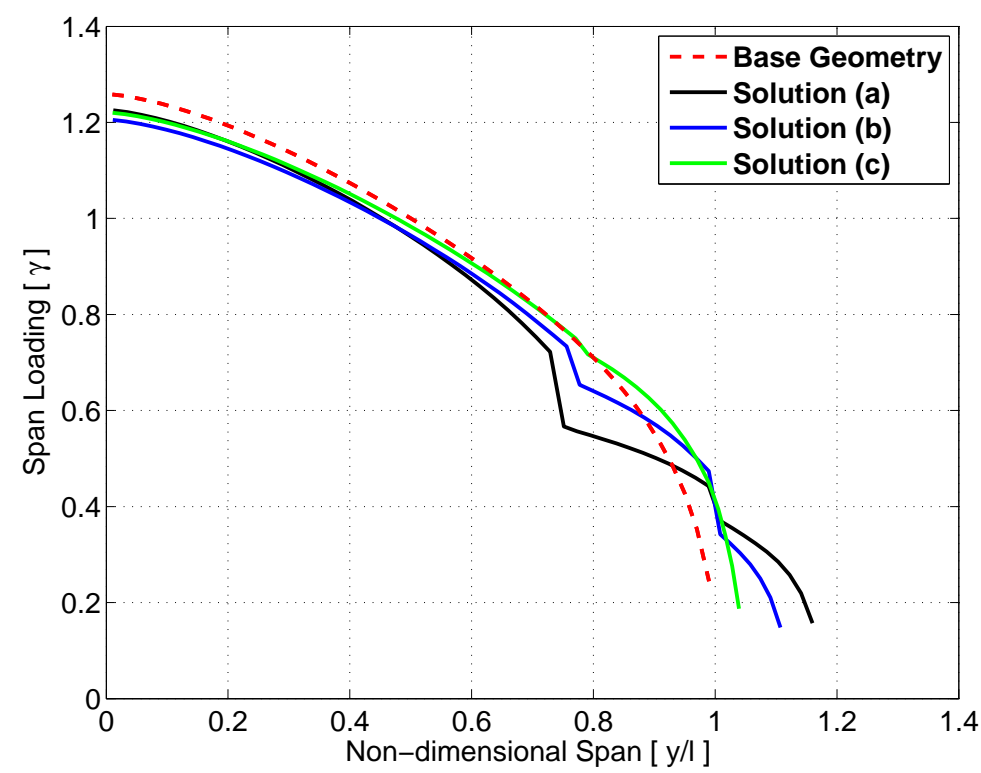

Figure 12. Span loading distributions for wing geometries shown in figure 11.

\section{Highly Non-planar Wing tip Optimisation}

The wing design problem is now extended to highly non-planar wing tip optimisation in which the outer $25 \%$ of the baseline geometry is optimised by the sGA. Up to three trapezoidal wing sections are optimised with the following design variables: span, taper ratio, dihedral, sweep, linear twist over each panel section, aerofoil camber for each section, and planform angle of attack. The sGA can also select aerofoil sections to readjust the camber between wing sections: NACA 65-210 ( $\left.A_{\text {ero } 1}\right)$ or NACA $0010\left(A_{\text {ero } 2}\right)$ may be selected. Additionally, the sGA is capable of inverting the camber and circulation direction of wing segments to download the wing: 0 resulting in positive camber, 1 in negative camber. The optimisation is presented as follows:

minimise: $D_{\text {ind }}$ and $D_{\text {para }}$
With respect to variables $\left\{\begin{array}{ll}0 \leq \Lambda_{i} \leq 45 & {[\text { deg }]} \\ 0.1 \leq \lambda_{i} \leq 1 & \\ 0 \leq \Gamma_{i} \leq 195 & {[\text { deg }]} \\ -10 \leq \theta_{i} \leq 10 & {[\text { deg }]} \\ 0 \leq b_{i} \leq 2 & {[\mathrm{~m}]} \\ 0 \leq \alpha_{i} \leq 12 & {[\text { deg }]} \\ \text { Aerofoil }=A_{\text {ero1 } 1}, A_{\text {ero } 2} \\ \text { Camber }=0,1\end{array} \quad\right.$ Subject to $\left\{\begin{array}{l}1-\frac{L}{L_{\text {ref }}} \leq 0 \\ \frac{b_{\text {ref }}}{h_{\text {wingtip }}}=0.25 \\ \Gamma_{\text {limit }}=45 \quad[\text { deg }] \\ M_{\text {root }} \leq m_{\text {limit }} M_{\text {root }} \\ \frac{b}{b_{\text {ref }}} \leq s_{\text {limit }} b_{\text {ref }}\end{array}\right.$

The sGA ran for 1000 generations with a population of 100; deemed to be sufficient based on convergence studies with the algorithm. The resulting Pareto fronts from two identical optimisations for the wing geometry constrained to the baseline wing span $\left(s_{\text {limit }}=1\right)$ and unconstrained RBM $\left(m_{\text {limit }} \rightarrow \infty\right)$ is shown 
in figure 13. The identified Pareto front has two distinct subsets of solutions, region (a) and region (b) highlighted in figure 13, between which exists a transitional region of solutions denoted region (c). Example wing configurations presented in figure 13 have been taken from the extremes of the Pareto and show the loading direction.

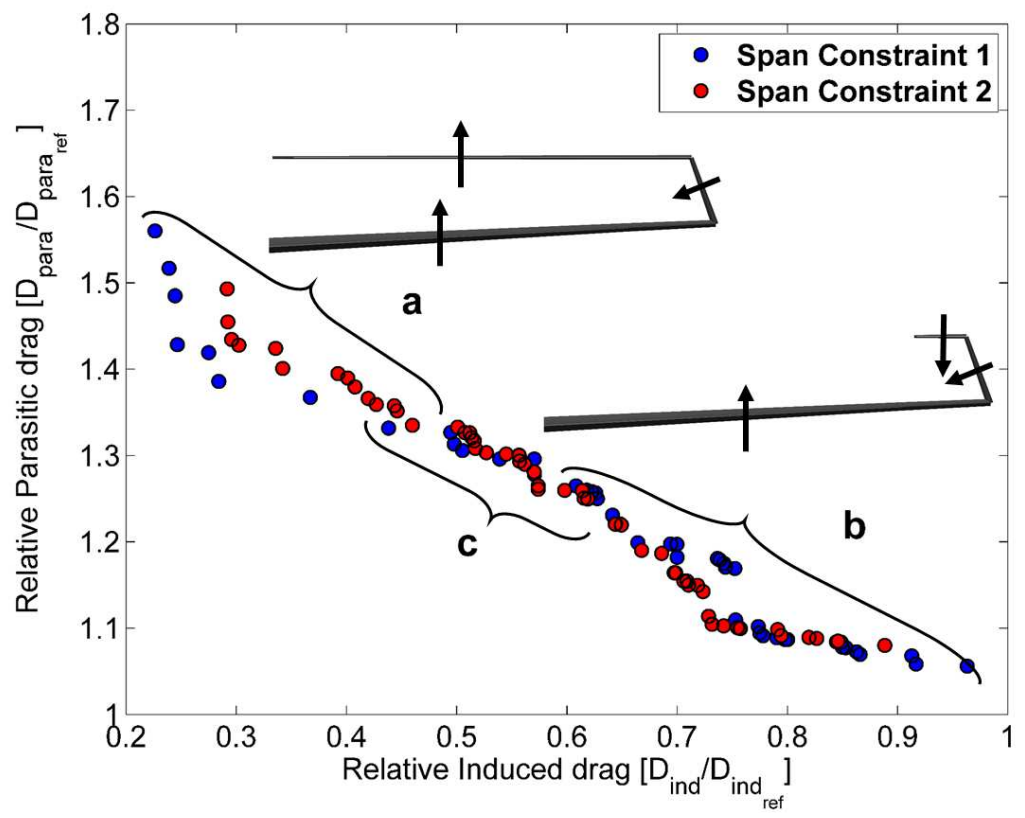

Figure 13. Pareto fronts of two identical optimisations for non-planar wings with no RBM constraints applied, only span constraints $\left(m_{\text {limit }} \rightarrow \infty, s_{\text {limit }}=1\right)$.

In figure 13 solutions achieving the greatest reduction of induced drag are found in region (a), $D_{\text {ind }} / D_{\text {inf }}$ ref $<$ 0.5. The winglet has gained a horizontal extension forming a closed box-wing with maximum allowable height and span. This is the same result expected from lifting line theory. The upper lifting surface is established with minimal taper and sweep to maximise lift. A significant portion of the total lift is contributed by the upper surface, allowing the planform to lower the angle of attack. The parasitic drag penalties incurred are largely offset by the increased induced efficiency: the greatest reduction in total drag was $21.6 \%$ where induced drag was reduced by $77.4 \%$ and parasitic drag increased by $55.9 \%$. As these solutions have very large horizontal extensions connected only at the wing tip, the RBM increased dramatically by up to $250 \%$.

In region (b), $D_{\text {ind }} / D_{\text {inf }}$ ref $>0.6$, the solutions mostly resemble vortex diffusers or C-wing configurations, while a few maintain highly swept winglets $\left(\Lambda>40^{\circ}\right)$. The $\mathrm{C}$-wing geometry has negative camber along the horizontal extension of the winglet while the winglet itself is uncambered with moderate twist along its span. Solutions achieved reductions of total drag up to $11.7 \%$, however demonstrate sensitivity to changes in the RBM dependant on the horizontal winglet extension. Some solutions reduce the RBM by $1-2 \%$ while others increase the RBM by up to $85 \%$. Intuitively, the horizontal extension is capable of tip loading the wing either negatively or positively depending on the configuration camber orientation, twist distribution, and planform angle of attack.

With the addition of RBM constraints with unconstrained span extensions, the optimiser identifies different solution fronts capable of greater reductions of induced drag relative to the rise in parasitic drag, as shown in figure 14. With no span constraint, the design of the non-planar wing/wing tip extends enabling better reduction of the induced drag while minimising gains in parasitic drag taking on a convex shaped Pareto front. When a span constraint is enforced (RBM 3 with span constraint) the front formed appears convex. It is found that the convex Pareto curvature follows the Pareto front seen at $\frac{D_{\text {ind }}}{D_{i} \text { dd }_{\text {ref }}} \approx 0.75$ in figure 13. This bend in the Pareto front is significant as it marks where along the front solutions have reached the span limiting constraint and now achieve further drag reductions by manipulation of the non-planar surfaces. This is seen to cause a more significant increase in the parasitic drag. 
The geometric representations of wing solutions (a), (b), and (c) highlighted in figure 14 are given in figure 15. All superior solutions identified by the sGA have winglets with uncambered aerofoil sections (NACA 0010), and the horizontal winglet extensions with negatively cambered sections (NACA 65-210). Solution (a) is identified as the optimal solution for RBM constraint 3 with span constraint $\left(m_{\text {limit }} \rightarrow\right.$ $\infty, s_{\text {limit }}=1$ ) with a $11.0 \%$ reduction in total drag with only an $9.2 \%$ increase in the root bending moment: this is consistent with previous span constrained results for $\mathrm{C}$-wings. Due to positive planform angle of attack the horizontal winglet extension is positively loaded, shown in figure 16. The negative camber and local twist minimise loading. The planform wing tip loading is reduced due to increased wing tip sweep which is found to offset the increased planform tip loading caused by the horizontal winglet extension.

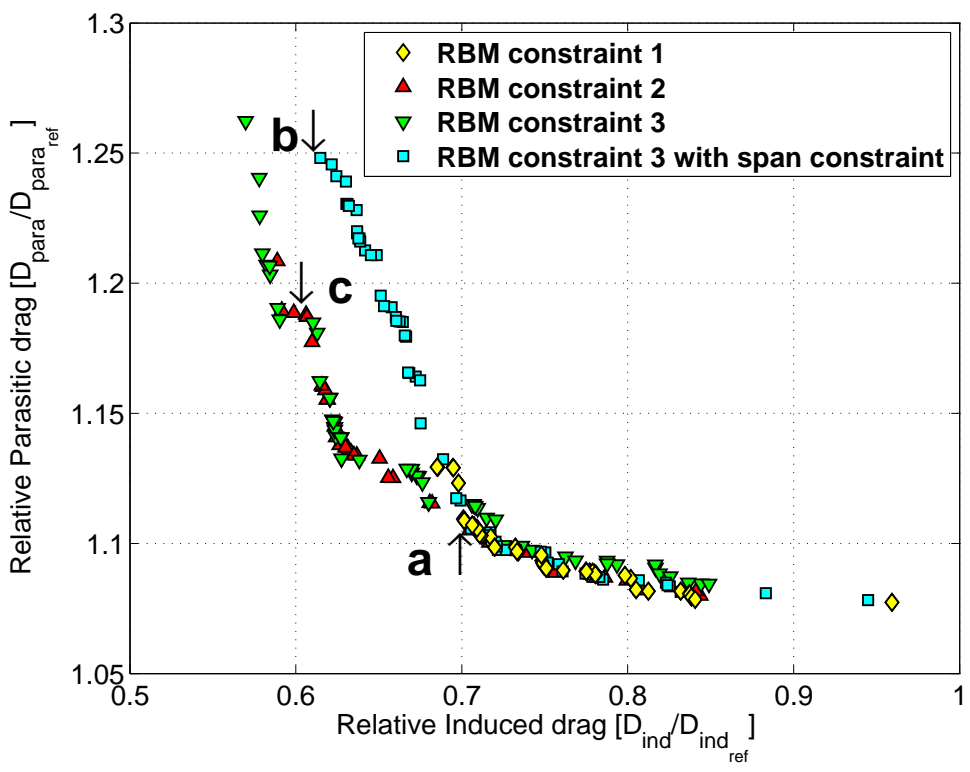

Figure 14. Pareto fronts of optimised non-planar wing tip geometry: RBM constraint $\mathbf{1}\left(m_{\text {limit }}=1.22, s_{\text {limit }} \rightarrow\right.$ $\infty)$; RBM constraint $2\left(m_{\text {limit }}=1.36, s_{\text {limit }} \rightarrow \infty\right)$; RBM constraint $\mathbf{3}\left(m_{\text {limit }}=1.52, s_{\text {limit }} \rightarrow \infty\right)$; and RBM constraint 3 with span constraint $\left(m_{\text {limit }}=1.52, s_{\text {limit }}=1.10\right)$

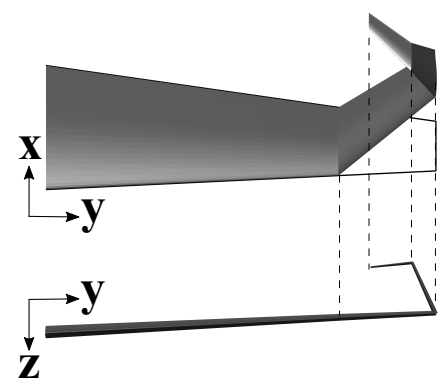

Solution (a)

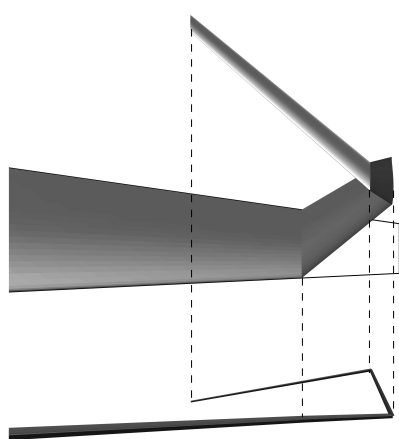

Solution (b)

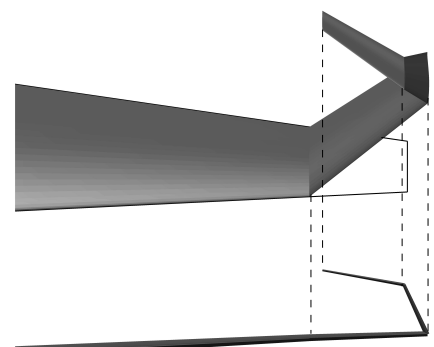

Solution (c)

Figure 15. Optimised non-planar configurations from Pareto fronts in figure 14. Baseline geometry outline shown.

Solution (b), identified at the upper extreme of Pareto RBM constraint 1, had a total drag saving of 9.7\%; relative to solution (a) total drag savings are reduced due the increased wetted area. The drop in loading at the wing tip of wing (b) is no longer enough to offset the increased tip loading due to the horizontal extension resulting in a RBM increase of $40 \%$. Wing solution (c) is the most superior solution with a $15.8 \%$ reduction in drag, partly due to a $5.1 \%$ span increase. Tip loading increased the RBM by $33.8 \%$. 


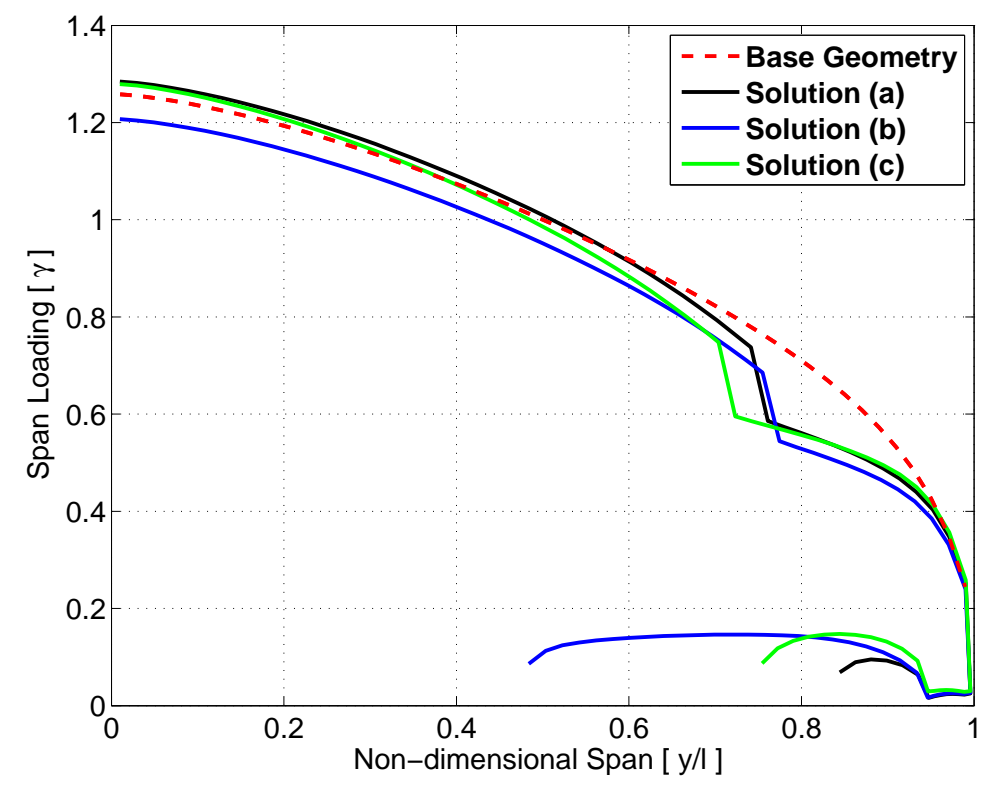

Figure 16. Span loading distributions for wing geometries shown in figure 15.

\section{Highly Non-planar Wing Optimisation with Static Margin Constraint}

Using optimisation constraints the winglet horizontal lifting surface (C-wing) can be exploited for longitudinal static stability, much like a horizontal stabiliser of an aircraft. To investigate the feasibility of maintaining a restoring pitching moment about the aerodynamic centre a static margin constraint has been added to the optimiser. Preliminary investigations using a central differencing analysis for each candidate solution using the VRM solver has been used to compute the pitch stiffness derivative, $C_{m_{\alpha}}$, and the lift-curve slope, $C_{L_{\alpha}}$. The static margin of the wing can then be estimated using: ${ }^{47}$

$$
C_{m_{\alpha}}=-K_{n} C_{L_{\alpha}} \cos (\alpha)
$$

In order to achieve a statically stable wing in pitch the optimiser is given full control of all four planform segments. Additional variables of centre of gravity location along the root chord line and variable root chord have also been added. The same constraints defined by the baseline geometry have been used. The optimisation is presented as follows:

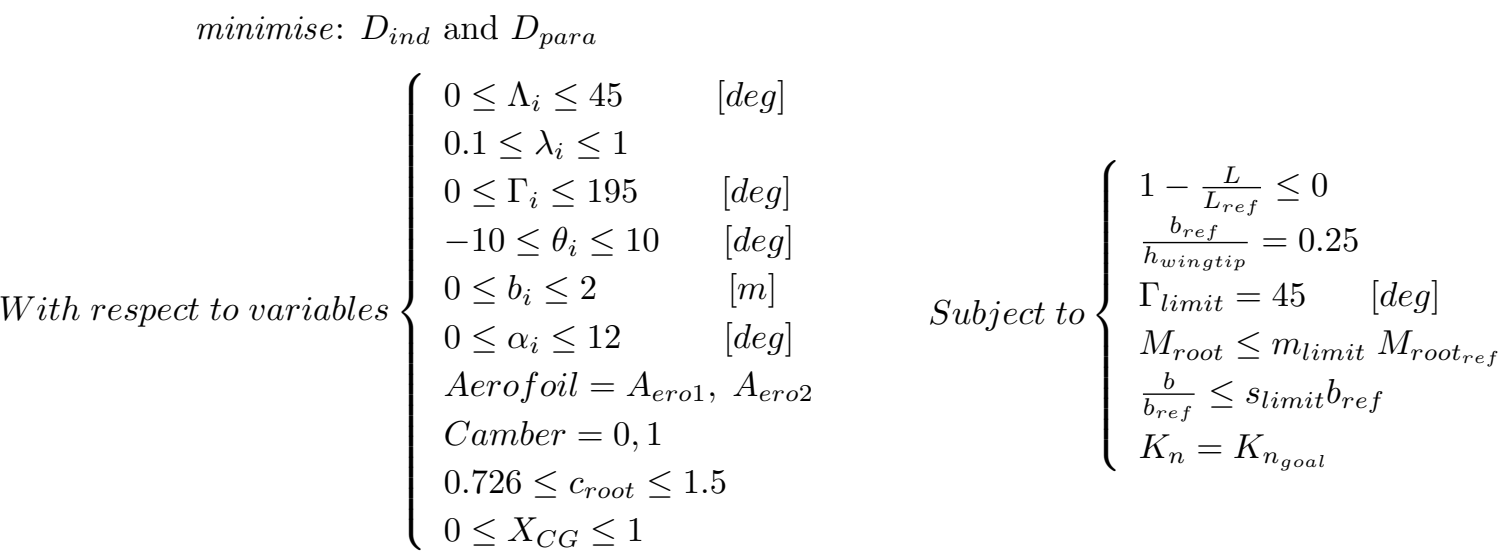

The sGA ran for 1000 generations with a population of 100 . However, convergence studies suggest more generations or a higher solution population should be used as further improvement can be expected. Using a static margin constraint of $K_{n}=+0.2$ with a $10 \%$ tolerance the resulting Pareto front of solutions is shown in figure 17. The wing solution that the sGA identified as the most superior solution in the population has been highlighted: with a total drag reduction of $15.2 \%$, an increase in RBM of $20.7 \%$ and a total span 
reduction of $2.5 \%$ relative to the baseline geometry for the same lift at a given angle of attack. The root chord increased by $12.8 \%$ which could be used to offset the gain in the root bending moment, however more in depth structural analysis would be required to quantify this.

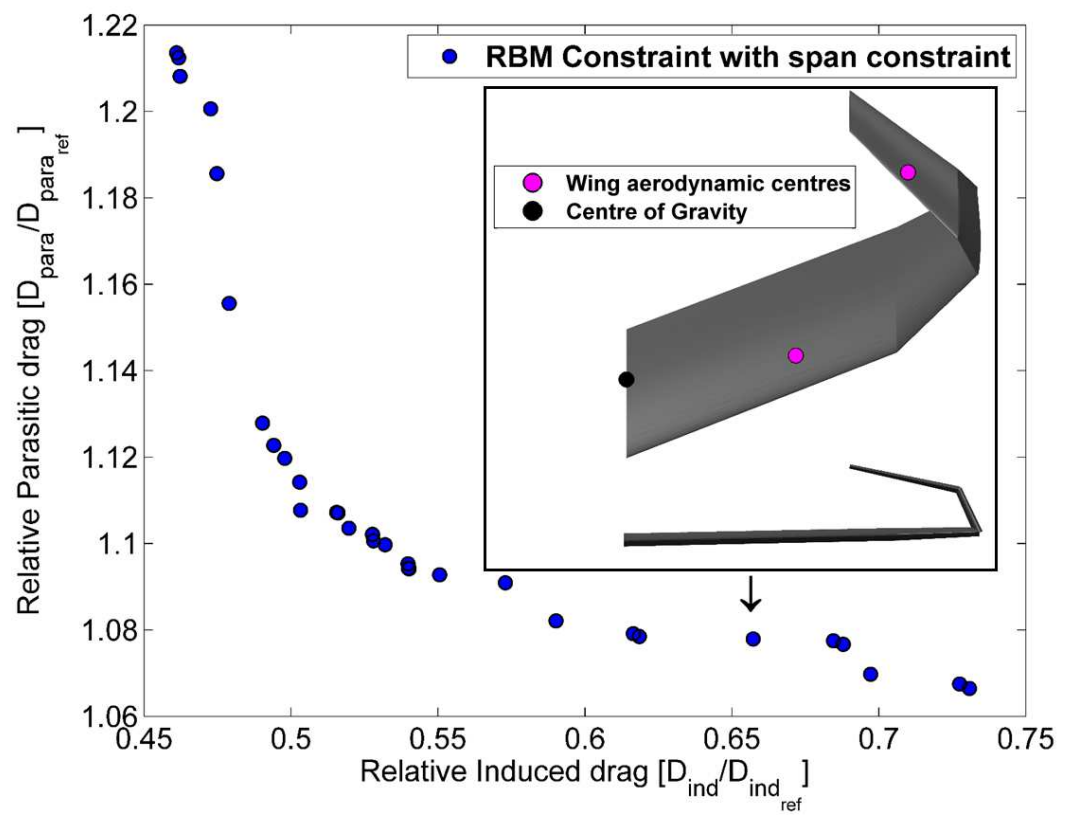

Figure 17. Pareto front for optimised non-planar wing geometry with static margin constraints $\left(m_{\text {limit }}=\right.$ $1.22, s_{\text {limit }}=1.10$, and $\left.K_{n}=+0.2 \pm 0.02\right)$.

For the wing solution highlighted in figure 17, the centre of gravity along the root chord line and the aerodynamic centres of the main wing lifting surface and the horizontal C-wing extension are shown. The main wing aerodynamic centre is positioned aft of the wing centre of gravity, with relative positions along the root chord at $0.80 C_{\text {root }}$ and $0.61 C_{\text {root }}$, respectively. The span loading of the wing at different angles of attack is shown in figure 18 .

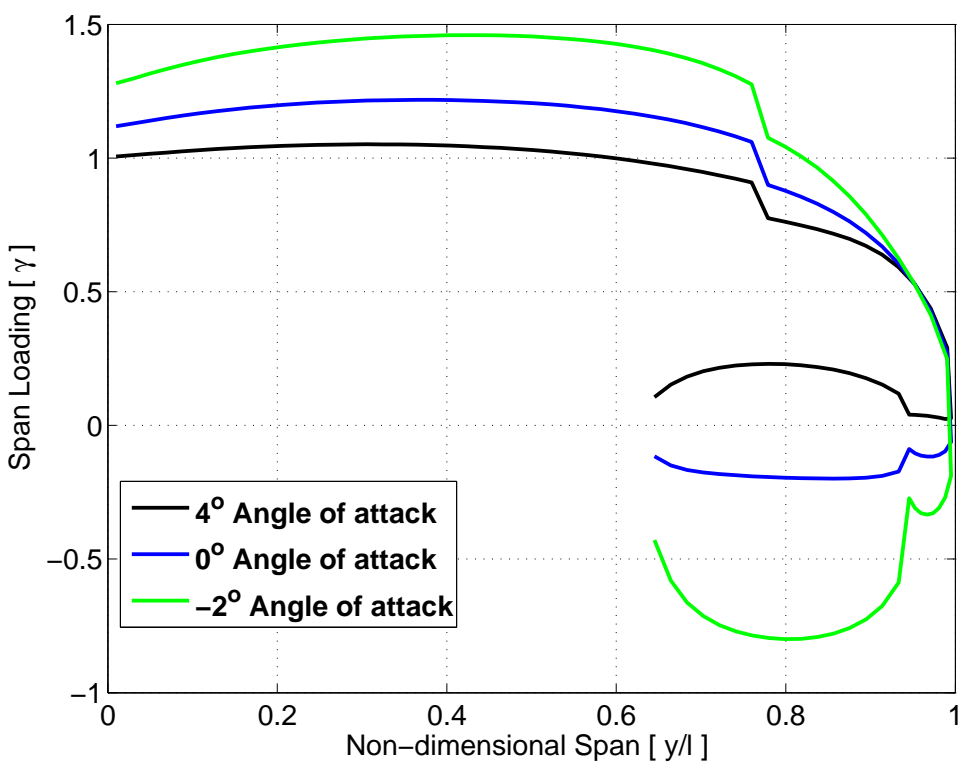

Figure 18. Span loading distributions for wing geometry shown in figure 17.

The relative amount of aerodynamic load over the span of the wing is expressed by the span loading parameter, hence the plots given for different angles of attack (changing the lift coefficient of the wing) are 
not directly comparable, but serve to demonstrate the changes in loading the wing experiences at a given angle of attack. At increased planform angles of attack the C-wing extension becomes positively loaded providing a nose down restoring moment about the centre of gravity. At a zero angle of attack the net moments about the centre of gravity are approximately zero. Reducing the angle of attack (effectively increasing the angle of attack of the horizontal extension) provides a nose up pitching moment. Beyond angles of attack less than two degrees the horizontal C-wing extension becomes heavily down-loaded, resulting in very strong nose up pitching moments which must overcome additional moments due to the forward centre of gravity location.

Although the wing appears to provide static stability based on simple numerical linearisation analysis, the horizontal tail volume coefficient is low, $\bar{V}_{h}=0.2143$. This suggests that the wing would have very poor longitudinal stability. The wing will also allow very little centre of gravity travel, this is expected as the sGA will have balanced relevant moment arms and lifting surfaces based on the centre of gravity location found in more favourable candidate solutions within the genetic algorithm population.

\section{Conclusion}

Non-planar aerodynamic optimisations have been carried out using a vortex ring potential flow solver integrated with a novel structured genetic algorithm (sGA). Methods used allow for quick force and moment assessment of how different non-planar configurations compare, and which design trends they follow dependant upon the parametrised design space and constraints imposed.

Optimisation of a simple wing extension to a fixed planform geometry suggests that wing extensions with relatively low dihedral offer better reductions in total drag when compared to wing extensions with significantly higher dihedral for the same root bending moment. It is also found that the induced drag is sensitive to small changes in tip extension sweep and dihedral.

When the optimisation design was extended to integrated wing tip/wing extension optimisation winglets incurred greater parasitic drag penalties more readily than tip extensions for similar gains in induced efficiency when the span is unconstrained. Winglets are found to obtain greater parasitic drag gain as their span is not as robustly suppressed by the RBM constraints. As a result, effectively unconstrained growth of the winglet length occurs. Due to the sectional Reynolds numbers of wing tip extensions being relatively lower than that of the wing significant gains in parasitic drag are made. Extensions of the wing tip with increasingly lower dihedral are more sensitively limited by the design root bending moment constraint minimising the parasitic drag gain. Pareto optimal results do show however that the induced efficiency of winglets is superior to tip extensions for equivalent total drag reduction.

For aerodynamic optimisation of highly non-planar wings, joined box-wings were found to be optimal in maximising induced efficiency when span constraints were enforced: reaching total drag reductions of up to $21.6 \%$. C-wing or vortex diffuser configurations under span constraints were found to be able to reduce drag by approximately $11 \%$. When the span was unconstrained $\mathrm{C}$-wings reduced total drag up to $15.8 \%$, also demonstrating sensitivity to changes in the root bending moment dependant upon the horizontal extension loading. Incorporating longitudinal static stability constraints into the optimisation of the C-wing identified Pareto optimal solution configurations with equivalent total drag savings (15.17\%). However, C-wing solutions with and without static stability constraints are not directly comparable.

Future work consists of the continued development of longitudinal static stability optimisation of Cwings. Additionally, the incorporation of aerostructural optimisation to analyse structural trade-offs will be implemented. This development will include optimisation of static aeroelastic deflections specifically to study the torsional deflections of $\mathrm{C}$-wings. Additionally an iterative force-free wake relaxation scheme to better compute the favourable fluid structure interactions of the wake produced by non-planar wings will be included.

\section{Acknowledgments}

The authors gratefully acknowledge the guidance and advice of Dr. Euan McGookin. 


\section{References}

${ }^{1}$ Kroo, I., "Non-planar Winng Concepts For Increased Aircraft Efficiency," VKI lectire series on Innivative Configurations and Advanced Concepts for Future Civil Aircraft, 2005, pp. 1-29.

${ }^{2}$ Khan, F., Krammer, P., and Scholz, D., "Preliminary Aerodynamic Investigation of Box-Wing Configurations Using Low Fidelity Codes," Deutscher Luft- und Raumfahrtkongress, DocumentID: 161308, 2010, pp. 313-327.

${ }^{3}$ Frediani, A., "The Prandtl Wing," VKI lectire series on Innivative Configurations and Advanced Concepts for Future Civil Aircraft, 2005, pp. 1-23.

${ }^{4}$ Schneider, W., "The Importance of Aerodynamics in the Development of Commercial Successful Transport Aircraft," Notes on Numerical Fluids Mechanics: Aerodynamic Drag Reduction Technologies, edited by P. Thiede, Vol. 76, Springer, 2000.

${ }^{5}$ Kennedy, G., Kenway, G., and Martins, J. R. R. A., "High Aspect Ratio Wing Design: Optimal Aerostructural Tradeoffs for the Next Generation of Materials," 52nd Aerospace Sciences Meeting, 2014, pp. 1-24.

${ }^{6}$ Morrison, S. A., "An Economic Analysis of Aircraft Design," Journal of Transport Economics, 1984, pp. $123-143$.

${ }^{7}$ Whitcomb, R. T., "A Design Approach and Selected Wind-Tunnel Results At High Subsonic Speeds For Wing-Tip Mounted Winglets," NASA Technical Note: TN D-8260, 1976.

${ }^{8}$ Spillman, J. J. and Allen, J. E., "The Use of Wing Tip Sails To Reduce Vortex Drag," 1977.

${ }^{9}$ Hackett, J. E., "US Patent Application for: "Vortex Diffuser," No. 4,190,219, filed 26 Feb. 1980," .

${ }^{10}$ Jupp, J. A. and Rees, P. H., "US Patent Application for:" Aircraft Wing and Winglet Arrangment," No. 4,714,215, filed 22 Feb. 1987,".

${ }^{11}$ Whitehouse, I., "Wing Tip Fences to Sharks : The Development of Airbus Winglets," Wing Aerodynamic Design, Airbus Operations Ltd., pp. 1-8.

${ }^{12}$ Heller, G., Maisel, M., and Kreuzer, P., "US Patent Application for: "Wing Tip Extension For A Wing," No. 6,772,615, filed 20 Feb. 2004," .

${ }^{13}$ Somerville, W. E., "US Patent Application for: "Flying Machine," No. 1,154,214, filed 4 Nov. 1910," .

${ }^{14}$ Hemke, P. E., "Drag of Wings with End Plates," NACA Technical Report TR-267, 1928.

${ }^{15}$ Mangler, W., "The Lift Distribution of Wings with End Plates," NACA Technical Memorandum TM-856, 1938.

${ }^{16}$ Riebe, J. M. and Watson, J. M., "The Effect of End Plates on Swept Wings at Low Speed," NACA Technical Report TN-2229, 1950.

${ }^{17}$ Jones, R. and Lasinski, T., "Effect of Winglets on the Induced Drag of Ideal Wing Shapes," NASA Techical Memorandum 81230, 1980.

${ }^{18}$ Kroo, I., "Design and Analysis of Optimally-Loaded Lifting Systems," AIAA, Vol. 84, No. No. 2507, 1984.

${ }^{19}$ Asai, K., "Theoretical Considerationa in The Aerodyanmic Effectivness of Winglets," Journal of Aircraft, Vol. 22, No. 7 , 1985, pp. 635-637.

${ }^{20}$ Heyson, H., Riebe, G., and Fulton, C., "Theoretical Parametric Study of the Relative Advantages of Winglets and Wing-Tip Extensions," NASA Technical Paper 1020, 1977.

${ }^{21}$ Terry, J., "Aerodynamic Charactaristics of Ring Wings: A Bibliography," Redstone Sceintific Information Center, Rept. RSIC-285, 1964.

${ }^{22}$ Miranda, L., "Boxplane Configuration for Conceptual Analysis and Initial Experimental Verification," Lochheed California Co., Rept. LR 25180, 1972.

${ }^{23}$ Wolkovitch, J., "The Joined Wing: An Overview," Journal of Aircraft, Vol. 23, 1986, pp. 161-178.

${ }^{24}$ Lee, H., Kim, Y., Park, G., Kolonay, R., Blair, M., and Canfield, R., "Nonlinear Response Structural Optimization of a Joined Wing Using Equivalent Loads," AIAA Journal, Vol. 44, No. 4, 2007, pp. 1302-1308.

${ }^{25}$ Kroo, I., Mcmasters, J., and Smith, S. C., "Highly Nonplanar Lifting Systems," Transportation Beyond 2000: Technologies Needed for Engineering Design, 1995.

${ }^{26}$ Mcmasters, J., Paisley, D., Hubert, R., Kroo, I., Bofah, K., and Sullivan, J., "Advanced Subsonic Configurations for Very Transport Airplanes Large," NASA Contractor Report 198351, 1996.

${ }^{27}$ Gage, P. J., New Approaches to Optimisation in Aerospace Conceptual Design, Phd. thesis, University of Stanford, 1994.

${ }^{28}$ Slingerland, R. and Verstraeten, J. G., "Drag Characteristics for Optimally Span-loaded Planar, Wingletted, and CWings," 2007, pp. 1-16.

${ }^{29}$ Ning, S. A. and Kroo, I., "Multidisciplinary Considerations in the Design of Wings and Wing Tip Devices," Journal of Aircraft, Vol. 47, No. 2, mar 2010, pp. 534-543.

${ }^{30}$ Jansen, P. W., Perez, R. E., and Martins, J. R. R. A., "Aerostructural Optimization of Nonplanar Lifting Surfaces," Journal of Aircraft, Vol. 47, No. 5, sep 2010, pp. 1490-1503.

${ }^{31}$ Wakayama, S. and Kroo, I., "Subsonic Wing Planform Design Using Multidisciplinary Optimization," Journal of Aircraft, Vol. 32, 1995, pp. 746-753.

${ }^{32}$ Obayashi, S. and Tsukahara, T., "Comparison of Optimization Algorithms for Aerodynamic Shape Design," AIAA Journal, Vol. 35, No. 8, 1997, pp. 1413-1415.

${ }^{33}$ Chiba, K., Obayashi, S., and Nakahashi, K., "High-Fidelity Multidisciplinary Design Optimization of Aerostructural Wing Shape for Regional Jet," 23rd AIAA Applied Aerodynamics Conference, AIAA 2005-5080, , No. June, 2005, pp. 621-635.

${ }^{34}$ Hashimoto, A., Jeong, S., and Obayashi, S., "Aerodynamic Optimization of Near-future High-wing Aircraft," Transactions of The Japan Society for Aeronautical and Space Sciences, Vol. 58, No. 2, 2015, pp. 73-82.

${ }^{35}$ Epstein, B., Peigin, S., and Tsach, S., "A new efficient technology of aerodynamic design based on CFD driven optimization," Aerospace Science and Technology, Vol. 10, No. 2, mar 2006, pp. 100-110.

${ }^{36} \mathrm{Li}$, D. and Hartmann, R., "Adjoint-Based Airfoil Optimisation With Discretization Error Control," International Journal for Numerical Methods in Fluids, Vol. 77, 2015, pp. 1-17. 
${ }^{37}$ Katz, J. and Plotkin, A., Low-Speed Aerodynamics, Cambridge University Press, Cambridge UK, 2 nd ed., 2001.

${ }^{38}$ Smith, S. C., "A Computational and Experimental Study of Nonlinear Aspects of Induced Drag: NASA Technical Paper 3598," Tech. rep., 1996.

${ }^{39}$ Drela, M., Flight Vehicle Aerodynamics, The MIT Press, Cambridge, MA, 2014.

${ }^{40}$ Sivells, J. C., "Experimental and Calculated Charactaristics of Three Wings of NACA 64-210 and 65-210 Airfoil Sections with and without 2 Degrees Washout," NACA TN-1422, 1947.

${ }^{41}$ Anderson, J., Fundmentals of Aerodynamics, McGraw-Hill, New York, 3rd ed., 2001.

${ }^{42}$ Zingg, D. W., Nemec, M., and Pulliam, T. H., "A comparative evaluation of genetic and gradient-based algorithms applied to aerodynamic optimization," European Journal of Computational Mechanics, Vol. 17, No. 1-2, mar 2008, pp. 103-126.

${ }^{43}$ Castillo, O., Melin, P., and Kacprzyk, J., Soft Computing for Hybrid Intelligent Systems, Springer, Berlin, Germany, 2008.

${ }^{44}$ Dasgupta, D. and Mcgregor, D. R., "sGA : A Structured Genetic Algorithm ." Tech. rep., University of Strathclyde, Glasgow, U.K.

${ }^{45}$ Deb, K., Pratap, A., Agarwal, S., and Meyarivan, T., "A fast and elitist multiobjective genetic algorithm: NSGA-II," IEEE Transactions on Evolutionary Computation, Vol. 6, No. 2, 2002, pp. 182-197.

${ }^{46}$ Kato, T., Shimoyama, K., and Obayashi, S., "Evolutionary Algorithm with Parallel Evaluation Strategy of Feasible and Infeasible Solutions Considering Total Constraint Violation," IEEE, Vol. 00, No. 978, 2015, pp. 986-993.

${ }^{47}$ Stevens, L. and Lewis, L., Aircraft Control and Simulation, John Wiley \& Sons, Inc., New Jersey, 2nd ed., 2003. 